\title{
Hemodynamic and Neuroendocrine Adaptations of the Preterm Lamb Left Ventricle to Acutely Increased Afterload
}

\author{
BARRY G. BAYLEN, YOUTARO AGATA, JAMES F. PADBURY, MACHIKO IKEGAMI, \\ ALAN H. JOBE, AND GEORGE C. EMMANOUILIDES \\ Department of Pediatrics, The Milton S. Hershey Medical Center, The Pennsylvania State University, Hershey, \\ Pennsylvania 17033; and The Perinatal Research Laboratories, Department of Pediatrics, Harbor UCLA \\ Medical Center, Torrance, California 90509
}

\begin{abstract}
The birth process is associated with dramatic alterations of left ventricular (LV) volume loading, pressure loading, and contractile state. The preterm $\mathrm{LV}$ has considerable volume loading reserve. We have assessed neuroendocrine and related hemodynamic responses of the preterm lamb $\mathrm{LV}$ at 0.8 gestation during acute pressure loading within the first 2-4 h after birth. We measured plasma catecholamines and hemodynamic and cineangiocardiographic parameters of $L V$ pump performance and contractility at basal levels and during rapid $L V$ pressure loading by partial balloon obstruction of the ascending aorta before and after propranolol. A relatively high level of propranolol ( $3 \mathrm{mg} / \mathrm{kg}$ ) was required to produce $\beta$-adrenoceptor blockade associated with reduction of heart rate and blood pressure, but after atrial pacing there was no detectable difference of basal $\mathrm{LV}$ pump performance or contractility at comparable heart rate, preload, and afterload. The LV pump performance was maintained and plasma catecholamines and $L V$ contractility were increased when aortic systolic pressure was augmented $60 \%$ over baseline. The increased contractile state at greater afterload was minimally blunted by propranolol. Thus the preterm $L V$ is relatively hypercontractile soon after birth and is capable of an integrated augmentation of pump performance and contractile state during pressure loading. These findings are relevant to the maintenance of adequate $L V$ performance and successful adaptation to the acute alterations of afterload associated with the transitional circulation at birth. (Pediatr Res 26: 336-342, 1989)
\end{abstract}

\section{Abbreviations}

EPI, epinephrine

ESV, end-systolic volume

$\mathrm{LV}$, left ventricle

mVCF, mean velocity circumferential fiber shortening

NOREPI, norepinephrine

PSP, peak systolic pressure

Various studies have suggested that the heart of the newborn animal functions at a relatively "hypercontractile" state in the

Received February 20, 1989; accepted June 6, 1989

Barry G. Baylen, M.D., Division of Pediatric Cardiology, The Milton S. Hershey Medical Center, P.O. Box 850, Hershey, PA 17033.

Supported by grants from the Harbor Collegium, Grants 590 GL03 and 676 IG from the American Heart Association, Greater Los Angeles Affiliate, and NIH Grants HD 12714, and HD 18014. first weeks immediately after birth (1-9). These speculations were supported by the findings that circulating catecholamines increased dramatically in preterm and term lambs, and even in human infants, shortly after birth $(10,11)$. Furthermore, the influence of circulating catecholamines upon the premature heart may be particularly acute as the developing myocardium is incompletely innervated $(3,6,12,13)$.

Recently it has become possible to evaluate cardiovascular performance in a stable exteriorized premature lamb preparation (14). Several investigators have evaluated the volume loading capacity of the preterm left ventricle in the surfactant treated lamb $(9,15,16)$. The purpose of this study was to assess contractility and performance of the preterm lamb left ventricle during pressure loading both before and after $\beta$-adrenoceptor blockade very shortly after birth at the time of maximum catecholamine release $(10,11)$.

\section{MATERIALS AND METHODS}

Animal preparation. Date-bred Western mixed-breed ewes obtained from a local supplier were sedated with ketamine $(5 \mathrm{mg} /$ $\mathrm{kg}$ ) and were delivered under spinal anesthesia by cesarean section of preterm lambs at $126-128$ days $(0.8)$ gestation. The lambs were tracheotomized before delivery under local anesthesia with $2 \%$ lidocaine hydrochloride and treated by endotracheal instillation of $15 \mathrm{~mL} 0.45 \mathrm{M} \mathrm{NaCl}$ containing natural sheep surfactant in suspension ( $50 \mathrm{mg}$ lipid per $\mathrm{kg}$ ) given before the first breath. The surfactant was isolated from the lung lavage of adult ewes (14). Each preterm lamb was then delivered and umbilical arterial cord blood sample was drawn for measurements of $\mathrm{pH}$ and arterial blood gases. The cord was clamped and cut; the animal was dried and was placed on a warming table and then ventilated with a pressure limited infant ventilator (Sechrist Industries, Anaheim, CA) at positive end-expiratory pressure of $2 \mathrm{~cm} \mathrm{H}_{2} \mathrm{O}$, peak-inspiratory pressure $20 \mathrm{~cm} \mathrm{H}_{2} \mathrm{O}$, and inspiratory time $1 \mathrm{~s}$ at a rate of $30 \mathrm{breaths} / \mathrm{min}$. A no. 5 French polyvinyl soft catheter was passed into an umbilical artery to the distal thoracic aorta, and each lamb was paralyzed with intravenous pancuronium bromide $(0.1 \mathrm{mg} / \mathrm{kg})$ in order to assure control of ventilation and comparable blood gases during the study period.

The ventilatory settings of peak-inspiratory pressure and endexpiratory pressure, rate, and inspiratory oxygen concentration were then adjusted to maintain arterial $\mathrm{PO}_{2} 50-80 \mathrm{~mm} \mathrm{Hg}, \mathrm{PCO}_{2}$ $30-45 \mathrm{~mm} \mathrm{Hg}$, and $\mathrm{pH} 7.35-7.45$. Arterial blood gases, $\mathrm{pH}$ and hematocrit were thereafter monitored at 20 - to 30 -min intervals during the study protocol.

Intravascular catheters were maintained patent by infusion of heparinized $5 \%$ dextrose-water $(15 \mathrm{~mL} / \mathrm{kg} / \mathrm{h})$. All blood losses 
were replaced with equivalent volumes of freshly collected, filtered anticoagulated, maternal blood. Rectal temperature was continuously monitored and maintained at $38-39^{\circ} \mathrm{C}$ using warming pads and surface heating lamps.

Sedation was achieved using $5 \mathrm{mg} / \mathrm{kg}$ ketamine; analgesia was accomplished using local infiltration of $2 \%$ lidocaine hydrochloride at cut down sites. Paralysis was maintained using periodic intravenous administration of Pancuronium. The tracheotomy and subsequent cut down sites were reinfiltrated with lidocaine hydrochloride hourly, and sedation was maintained for the duration of the protocol with ketamine at hourly intervals. Procedures and protocol were reviewed and carried out in accordance with the standards of the American Physiological Society under local institutional animal use committee guidelines.

Catheter placement. The animals were catheterized under local anesthesia ( $2 \%$ lidocaine hydrochloride) through small external cutdowns using fluoroscopy and pressure monitoring (Figs. 1 and 2). The tracheotomy incision was extended laterally to expose the right external jugular vein and carotid artery. A right inguinal cutdown was performed to expose the femoral artery and vein. Two catheters were passed into the right external jugular vein; a no. 4F balloon angiography catheter was passed into the main pulmonary artery, and a no. $5 \mathrm{~F}$ J-shaped bipolar electrode pacing catheter into the right atrium (Fig. 1). A no. 4F flow directed flexible balloon angiography catheter (Edwards Laboratories, Santa Ana, CA) was passed into the umbilical vein (or when the ductus venosus was constricted, into the right femoral vein) through the foramen ovale, and with the balloon inflated the catheter was advanced to the apex of the left ventricle. A no. 3F polyvinyl catheter was advanced into the second umbilical artery (or right femoral artery) to the ascending aorta just above the aortic valve. Finally, a no. $5 \mathrm{~F}$ balloon atrial septostomy catheter (Edwards Laboratories) was advanced retrograde from the right carotid artery to the ascending aorta just above the aortic valve and positioned just distal to the end hole

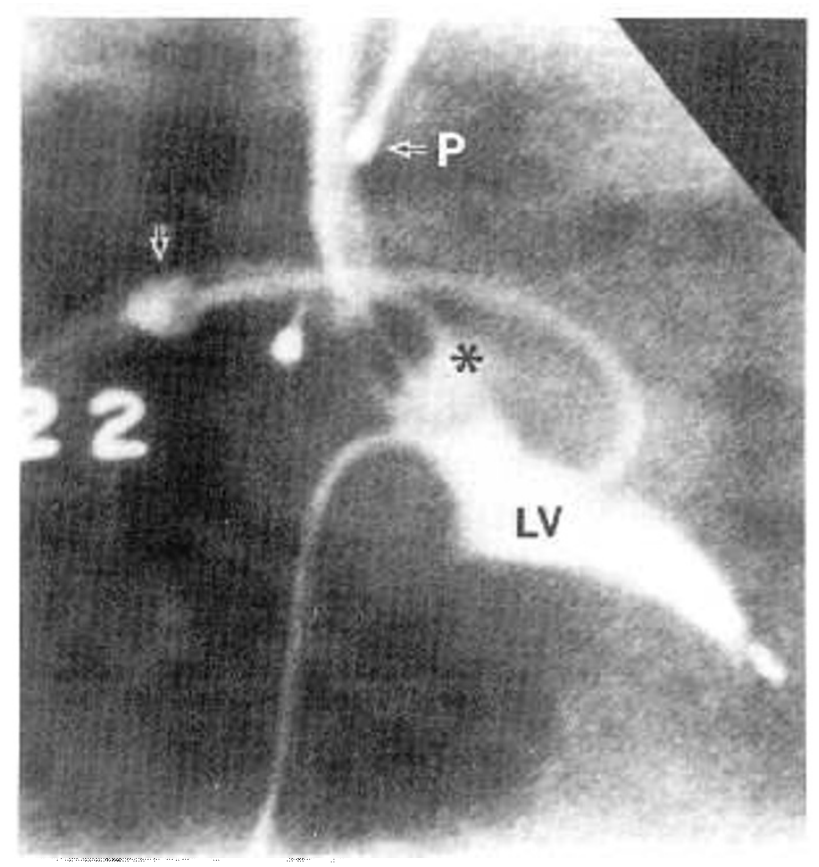

Fig. 1. Single frame of cine left ventriculogram from the preterm lamb preparation. Venous catheters are advanced to right atrium and pulmonary artery. A bipolar pacing catheter $(P)$ is in the right atrium and radiopaque inflated balloon catheter occludes the lumen of the ductus arteriosus (vertical arrow). A no. 4F balloon angiography catheter is in the left ventricle ( $L V$ ). A catheter balloon (asterisk) is inflated within the ascending aorta and contrast agent flows around the balloon to the distal aortic arch. of the polyvinyl catheter, such that the latter would measure ascending aortic pressure proximal to the balloon (Figs. 1 and 2 ). The position of the catheters was confirmed by fluoroscopy and pressure monitoring such that inflation of the balloon permitted recordings of ascending and descending aortic pressures, proximal and distal to the obstructing balloon. The catheter in the pulmonary artery was advanced into the ductus arteriosus lumen, and the balloon was then inflated to occlude the ductus arteriosus. The catheter was secured, and occlusion of the ductus during the duration of the protocol was confirmed by pressure monitoring and fluoroscopy $(9,16)$.

Measurements and calculations. The hemodynamic pressures were continuously monitored and recorded with a multichannel photographic recorder (Hewlett-Packard Co., Palo Alto, CA) at paper speed $100 \mathrm{~mm} / \mathrm{s}$. Transducers were calibrated with a mercury manometer and referred to zero position at the midthoracic level (lateral decubitus position) before each series of measurements. LV-PSP was measured from the maximum excursion of the plateau portion of the LV systolic pressure wave on rapid paper speed recordings. The first derivative of the systemic arterial pressure wave form was used for timing purposes and measured with a Hewlett-Packard derivative preamplifier. Heart rates were calculated from five successive heart beats. $\mathrm{mVCF}$ was calculated from the standard equation using the angiographically derived LV semiminor axis and the LV ejection time measured from the aortic pressure tracing (17). Comparison of $\mathrm{mVCF}$ at different intervals during the protocol was at essentially equivalent heart rate and, consequently, LV ejection time was not corrected for heart rate (17).

Left ventriculograms were obtained from lambs lying in the left lateral decubitus position using $1 \mathrm{~mL} / \mathrm{kg}$ IODOHEXOL-350 (Winthrop-Breon, New York, NY) injected through the LV angiography catheter. Cineangiocardiograms were recorded on $16 \mathrm{~mm}$ film at a rate of 60 frames/s (Fig. 1). Left ventricular volumes were calculated by the single plane area-length method from three to five projected frames as previously described (18, 19).

Analytical techniques. Blood samples for catecholamine measurements $(1 \mathrm{~mL})$ were placed immediately into chilled tubes containing EGTA and reduced glutathione. The plasma was rapidly separated, frozen, and stored for assay. The plasma catecholamine concentrations were determined by a radioenzymatic assay sensitive to $10-20 \mathrm{pg} / \mathrm{mL}$ NOREPI and EPI (11). Blood gases were measured on a calibrated radiometer blood gas instrument.

Experimental protocol. We evaluated pump performance and contractility by measuring left ventricular performance at varying afterload, i.e. at baseline, and then during inflation of a catheter balloon in the ascending aorta. After a recovery period of approximately $1 \mathrm{~h}$, the same experimental protocol was then repeated after intravenous administration of propranolol. The preliminary studies to develop a method for varying LV-PSP in a predictable manner, and the protocol for $\beta$-adrenoceptor blockade are described below.

Approximately $50 \mathrm{~min}$ after recovery from catheterization (approximately $2 \mathrm{~h}$ postnatal age) baseline hemodynamics and blood samples were obtained. Blood samples were obtained for hematocrit, arterial blood gas, $\mathrm{pH}$, and plasma catecholamines; then baseline left ventriculogram was performed. Ten min later, ascending aortic balloon inflation was accomplished to raise LVPSP $60 \%$ over baseline, and blood sampling, hemodynamic measurements, and left ventriculogram were obtained during the 3rd-5th min of balloon inflation.

The catheter balloon was then deflated, and after a 60 -min recovery period, a second series of measurements was obtained before and after infusion of propranolol as follows: preinfusion arterial blood gases hemodynamic measurements and blood plasma samples for catecholamines were obtained. After establishing $\beta$-adrenoceptor blockade with propranolol, the heart rate and blood pressure measurements were again recorded. Then 


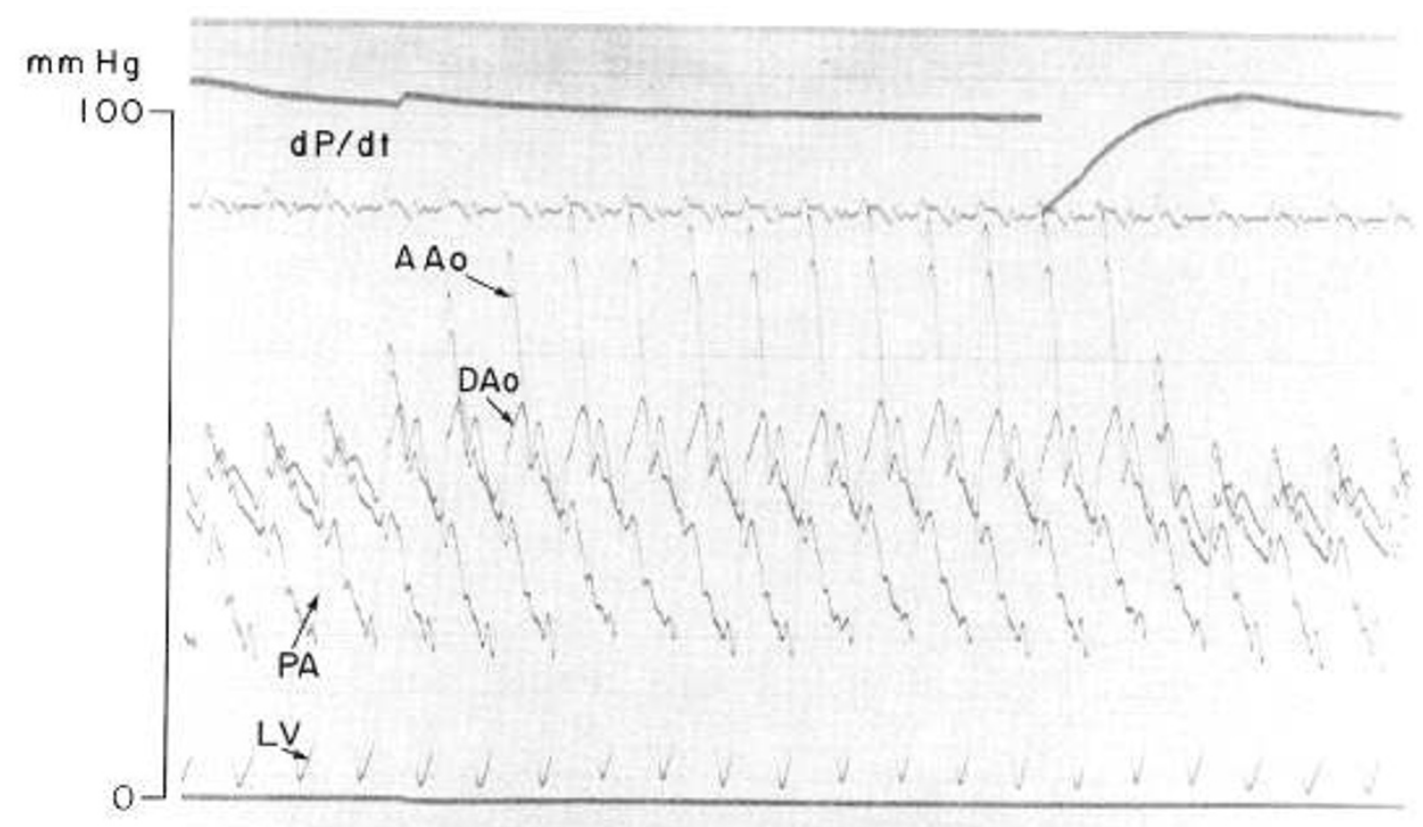

Fig. 2. Demonstration of instantaneous hemodynamic pressures and first derivative of aortic pressure $(d p / d t)$ recorded at slow paper speeds during ascending aortic balloon inflation (first electrical deflection) and deflation (second deflection). Pressures include ascending aorta (AAO) proximal to balloon, descending aorta $(D A o)$ distal to the balloon, left ventricular $(L V)$, and pulmonary artery $(P A)$ pressures with the ductus arteriosus occluded. Note during inflation LV and proximal AAo systolic pressures increase to $80 \mathrm{~mm} \mathrm{Hg}$, approximately $60 \%$ over baseline (50 $\mathrm{mm} \mathrm{Hg}$ ). There is a pressure gradient between AAo and DAo, and DAo pressure increases slightly.

right atrial pacing was initiated such that heart rate was nearly equal to that recorded during the first baseline study. Blood sampling, hemodynamic measurements and left ventriculogram were again obtained just before and during balloon inflation as described above.

Preliminary studies. In preliminary studies of preterm lambs, we found that a catheter balloon inflated to nearly completely obstruct the descending thoracic aorta produced a minimal and variable increase of LV-PSP. Consequently, we chose to inflate the catheter balloon in the ascending aorta, and the method produced the desired increase of LV-PSP. A relatively inflexible catheter was required to maintain the position of the inflated balloon in the ascending aorta. Cine left ventriculograms demonstrated that contrast agent flowed freely around the balloon into the distal ascending aorta (Fig. 1). Preliminary hemodynamic and angiographic studies demonstrated that preterm lambs consistently tolerated an increase of LV-PSP up to $60 \%$ over baseline for 3-5 min without apparent deterioration of $\mathrm{LV}$ ejection performance, significant elevation of end-diastolic pressure, mitral or aortic valvular insufficiency or deterioration of arterial blood gases. Consequently, for all subsequent studies in the protocol we chose to compare baseline hemodynamic values with the hemodynamic values measured after inflating the balloon for 3-5 min such that LV-PSP was increased $60 \%$ over baseline LV-PSP, that is at comparable pressure levels for each animal.

As mentioned, baseline hemodynamic values and values obtained during balloon inflation were then repeated after intravenous bolus and maintenance infusion of propranolol. The propranolol and isoproterenol were weighed and freshly prepared from fresh reagents (Sigma Chemical Co., St. Louis, MO). In these preliminary studies, using stepwise $1 \mathrm{mg} / \mathrm{kg}$ boluses, we found that a dose of $3 \mathrm{mg} / \mathrm{kg}$ propranolol was required to consistently establish $\beta$-adrenoceptor blockade in the preterm lambs at approximately 2 to $3 \mathrm{~h}$ postnatal age. $\beta$-Adrenoceptor blockade was defined as a decrement to minimum heart rate that remained unchanged during immediate challenge with isoproterenol infused at $0.1 \mu \mathrm{g} / \mathrm{kg} / \mathrm{min}$ for $3 \mathrm{~min}$. Consequently, for all studies a total of $3 \mathrm{mg} / \mathrm{kg}$ propranolol was infused intravenously at a rate of $1 \mathrm{mg} / \mathrm{kg} / \mathrm{min}$. The $\beta$-adrenoceptor blockade was then confirmed (as defined above) in all cases by immediate infusion of isoproterenol, $0.1 \mu \mathrm{g} / \mathrm{kg} / \mathrm{min}$ intravenously for $3 \mathrm{~min}$. $\beta$ Adrenoceptor blockade was then maintained by continuous infusion of $0.5 \mu \mathrm{g} / \mathrm{kg} / \mathrm{min}$, a dose previously demonstrated to maintain blockade (20).

Statistics. Comparisons of data from the same animals before and after balloon inflations pre- and postpropranolol were made using a one-way analysis of variance for multiple comparisons between group means. Student-Newman-Keuls test was used to test for significant differences between the individual group means. To provide homogeneity of variances, all catecholamine values were log transformed before statistical analyses and are presented as geometric mean \pm SE. A $p<0.05$ (two-tailed) was accepted as demonstrating a significant difference between group means. All data are expressed as mean \pm SE.

\section{RESULTS}

The data are presented for nine lambs that were stable throughout the delivery, catheterization and study protocol. Four additional lambs were eliminated from the study after sustained hypotension and acidemia, prolonged cardiac arrhythmia, LV dysfunction and mitral valvular incompetence (during balloon inflation), or cardiac trauma (postmortem). In the following discussion, data obtained before balloon inflations are referred to as "baseline" and data obtained during inflations are designated "inflation."

Blood gases. Table 1 demonstrates the arterial $\mathrm{pH}$ and blood gas data obtained at baseline and during the balloon inflations, before and after administration of propranolol. The initial baseline $\mathrm{pH}$ and blood gases before balloon were obtained beginning at $120 \pm 8 \mathrm{SE}$ min postnatal age, and the second baseline data after recovery and just before propranolol were obtained at 195 $\pm 9 \mathrm{~min}$ of age. The initial baseline arterial pH (7.40), $\mathrm{PO}_{2}(66$ $\mathrm{mm} \mathrm{Hg})$, and $\mathrm{PCO}_{2}(34 \mathrm{~mm} \mathrm{Hg})$ remained acceptable and did not change significantly during the period of study (Table 1). 
During the balloon inflations arterial $\mathrm{PO}_{2}$ increased significantly $(p<0.01)$. Arterial $\mathrm{pH}$ and $\mathrm{PCO}_{2}$ did not change significantly during the sustained balloon inflations.

Hemodynamic data. The mean baseline heart rate was $152 \pm$ 8 beats/min before propranolol, and decreased significantly to $123 \pm 7$ beats/min $(p<0.01)$ after intravenous propranolol and isoproterenol challenge. The aortic mean blood pressure decreased significantly after propranolol and before pacing from $41 \pm 3$ to $37 \pm 2 \mathrm{~mm} \mathrm{Hg}(p<0.05)$.

Figures 3 through 5 demonstrate the hemodynamic responses at baseline and during balloon inflations before and after intravenous propranolol administration. For the remainder of the discussion, baseline and inflation data after propranolol administration are recorded while pacing the right atrium at the same heart rate measured during the initial (prepropranolol) baseline. Heart rates increased slightly but not significantly during inflation before propranolol. As expected, after atrial pacing, heart rates calculated at baseline and during inflation were essentially equivalent to the initial baseline rate $(152 \pm 8$ beats $/ \mathrm{min}$ ) (Fig. 3). "Downstream" total systemic vascular resistance was $0.37 \pm$ $0.01 \mathrm{~mm} \mathrm{Hg} / \mathrm{mL} / \mathrm{kg} / \mathrm{min}$ before inflation and did not change significantly during inflation $(0.45 \pm 0.01)$ or after propranolol.

The baseline left ventricular peak systolic and aortic systolic pressure $(61 \pm 4 \mathrm{~mm} \mathrm{Hg})$, did not change significantly after propranolol administration and pacing (Fig. 4). Similarly, there was no difference comparing inflation versus inflation LV-PSP before and after propranolol. As expected, the LV-PSP increased significantly during inflation before $(95 \pm 4 \mathrm{~mm} \mathrm{Hg})$ and after propranolol. Thus, mean baseline and inflation heart rates and baseline and inflation LV-PSP were comparable during the preand postpropranolol studies.

Table 1. Arterial pH, blood gases at baseline and during balloon inflation before and after propranolol

\begin{tabular}{cccccc}
\hline & \multicolumn{2}{c}{ Prepropranolol } & & \multicolumn{2}{c}{ Propranolol } \\
\cline { 2 - 3 } \cline { 5 - 5 } Age & Baseline & & & Baseline & \\
& $(120 \pm 24)^{*}$ & Inflation & & $(195 \pm 28)$ & Inflation \\
\hline $\mathrm{pH}$ & $7.40 \pm 0.02$ & $7.41 \pm 0.03$ & & $7.36 \pm 0.03$ & $7.39 \pm 0.04$ \\
$\mathrm{PO}_{2}$ & $67 \pm 6$ & $116 \pm 21 \dagger$ & & $74 \pm 9$ & $140 \pm 22 \dagger$ \\
$\mathrm{PCO}_{2}$ & $34 \pm 1$ & $33 \pm 2$ & $37 \pm 3$ & $33 \pm 4$ \\
\hline
\end{tabular}

* Postnatal age - min (mean $\pm \mathrm{SE}$ ).

$\dagger p<0.01$, ANOVA-SNK base versus inflation.
HEART RATE

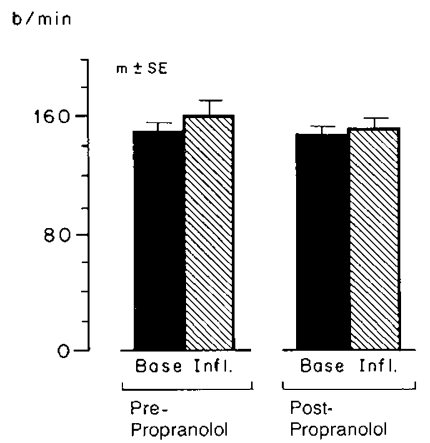

LEFT VENTRICULAR END DIASTOLIC VOLUME

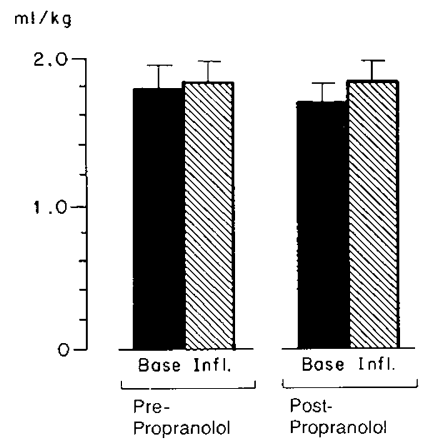

Fig. 3. Histograms of heart rates and LV end-diastolic volumes at baseline (Base) and during aortic balloon inflations (Infl.) before and after propranolol administration (right atrial pacing was initiated after propranolol). There was no significant change of the baseline heart rate during inflation either before or after propranolol. There were no significant differences comparing baseline versus baseline heart rates or inflation versus inflation heart rates before and after propranolol (analysis of variance-Student-Newman-Keuls test $=$ NS). Similarly, LV end-diastolic volume did not change during the studies.

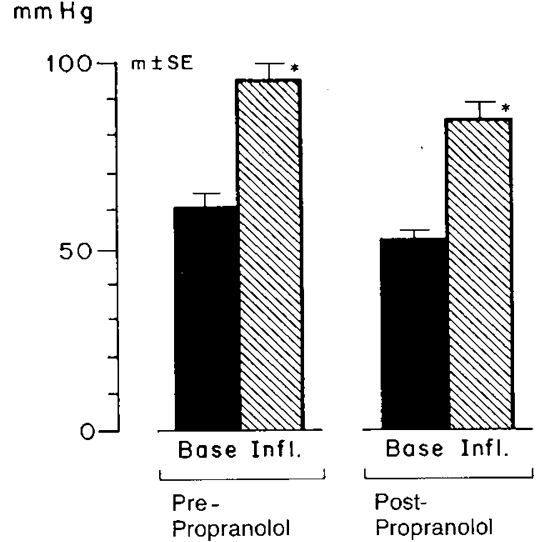

Fig. 4. Histograms of LV-PSP at baseline and during inflation before and after propranolol. The LV-PSP increased significantly during inflation* both before and after propranolol. There was no significant difference comparing baseline versus baseline LVPSP or inflation versus inflation LVPSP before or after propranolol [analysis of variance Student-Newman-Keuls test (ANOVA-SNK = NS)]. * ANOVA-SNK; $p$ $<0.001)$.
EJECTION FRACTION

EF \%

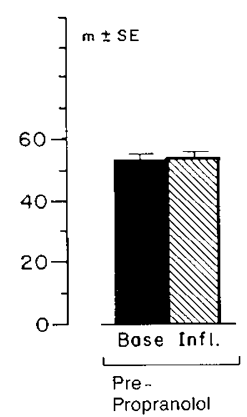

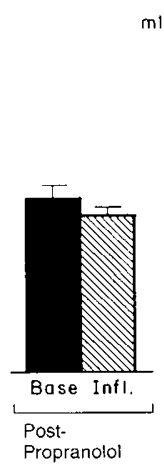

LEFT VENTRICULAR CARDIAC OUTPUT

$\mathrm{m} 1 / \mathrm{kg} / \mathrm{min}$

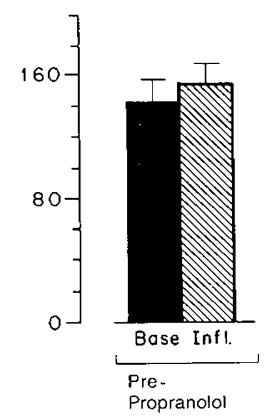

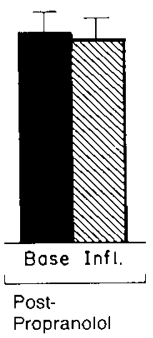

Fig. 5. Histograms of ejection fraction $(E F \%)$ and LV cardiac output at baseline and during inflation before and after propranolol. There was no significant change of baseline EF\% during inflations or of LV cardiac output during inflation either before or after propranolol. There were no differences comparing baseline versus baseline or inflation versus inflation $\mathrm{EF} \%$ before or after propranolol. Similarly, there were no differences comparing baseline versus baseline or inflation versus inflation cardiac output before or after propranolol.

The mean LV end-diastolic volumes $(1.77 \pm 0.15 \mathrm{~mL} / \mathrm{kg}) \mathrm{did}$ not change significantly when comparing baseline versus baseline or inflation versus inflation before and after propranolol administration. Inflation was not associated with significant change of LV end-diastolic volume before or after propranolol (Fig. 3). Consequently, indices of LV pump performance and contractility were measured at similar preload (end-diastolic volume), afterload (peak systolic pressure), and heart rate before and after propranolol at baselines and during balloon inflations.

The LV pumping performance as represented by ejection fraction and cardiac output was stable and acceptable during the protocol. Baseline LV ejection fractions $(53 \pm 2 \%)$ did not change significantly during inflations either before or after propranolol. In addition, for ejection fraction, there was no significant difference comparing baseline versus baseline or inflation versus inflation before and after propranolol (Fig. 5). Similarly, the baseline LV cardiac output $(147 \pm 15 \mathrm{~mL} / \mathrm{kg} / \mathrm{min})$ did not change significantly during inflations either before or after propranolol, and there was no significant difference comparing baseline versus 
baseline or inflation versus inflation cardiac output before and after propranolol.

The baseline LV contractility as represented by the $\mathrm{mVCF}$ was $1.81 \pm 0.14 \mathrm{circ} / \mathrm{s}$, and did not change significantly after propranolol $(2.16 \pm 0.25 \mathrm{circ} / \mathrm{s})$ at comparable heart rate (paced), LV end-diastolic volume and systemic arterial pressure. The contractility of the LV was also expressed as the ratios of LVPSP:ESV before and after inflation pre- and postpropranolol (21) (Table 2). There was no significant difference comparing baseline LV-PSP:ESV $\left(76 \pm 4 \mathrm{~mm} \mathrm{Hg}-\mathrm{ml}^{-1}-\mathrm{kg}^{-1}\right.$ ) versus baseline after propranolol. The LV-PSP:ESV ratios increased significantly during inflation before $(119 \pm 9)$ and after $(97 \pm 9)$ propranolol. However, inflation LV-PSP:ESV after propranolol was somewhat less than inflation LV-PSP:ESV before propranolol. The data suggest that increased LV contractility was associated with inflation both before and after propranolol, however, the increase was blunted after propranolol administration (21).

Table 2 demonstrates the plasma EPI and NOREPI levels at baseline and during the balloon inflations before and after propranolol. In all studies the baseline catecholamine levels were obtained just before propranolol administration as well as 5-10 min after propranolol, and these levels did not differ significantly. Consequently, only the postpropranolol catecholamine levels are reported as "baseline" in Table 2. The baseline plasma EPI level was $351 \pm 120 \mathrm{pg} / \mathrm{mL}$ and NOREPI was $366 \pm 125 \mathrm{pg} / \mathrm{mL}$; the baseline levels did not change after propranolol, and these values were comparable with reported levels for lambs of similar gestational and postnatal age (11). The plasma EPI levels increased significantly during the balloon inflations, however, the increase was marginally significant before the administration of propranolol ( $p<0.05$, one-tailed). Plasma NOREPI levels tended to increase during the balloon inflations, but this increase was only significant before the administration of propranolol (Table 2).

\section{DISCUSSION}

Studies of the transitional circulation in the intact preparation suggested that the heart of the full-term animal functions at a relatively high level of performance soon after birth (1). Subsequent studies demonstrated that the intact preterm lamb LV at 0.8 gestation possesses considerable volume loading capacity, and the volume loading reserve of the LV was similar to that of the term animal $(9,15,16)$. Furthermore, the augmented volume loading response in preterm lambs with patent ductus arteriosus was also relatively dependent on contractile state and was depressed during $\beta$-adrenoceptor blockade (8). To our knowledge the performance and contractility of the intact preterm LV during acute pressure loading have not been previously described.

We have evaluated the LV performance during pressure loading by partially obstructing the ascending aorta. The left ventricular performance, arterial $\mathrm{pH}$, and blood gases were stable and

Table 2. Plasma catecholamines and ratio of left ventricular PSP:ESV at baseline and during balloon inflation before and after propranolol (mean $\pm S E$ )

\begin{tabular}{lccccc}
\hline & \multicolumn{2}{c}{ Prepropranolol } & & \multicolumn{2}{c}{ Propranolol } \\
\cline { 2 - 3 } \cline { 5 - 5 } & Baseline & Inflation & & Baseline & Inflation \\
\hline NOREPI (pg/mL) & $366 \pm 125$ & $1022 \pm 275^{*}$ & $258 \pm 96$ & $562 \pm 142$ \\
EPI (pg/mL) & $351 \pm 120$ & $1916 \pm 775 \dagger$ & $248 \pm 119$ & $1200 \pm 320 \ddagger$ \\
LV-PSP:ESV & $76 \pm 4$ & $119 \pm 9 \S$ & $72 \pm 5$ & $97 \pm 9 \S \|$ \\
& & & & \\
$\left(\mathrm{mm} \mathrm{Hg} / \mathrm{mL}^{-1} /\right.$ & & & & \\
$\left.\mathrm{kg}^{-1}\right)$ & & &
\end{tabular}

${ }^{*} p<0.01$ base versus inflation.

$\dagger p<0.05$ base versus inflation (one-tail).

$\ddagger p<0.01$ base versus inflation.

$\S p<0.01$ base versus inflation.

$\| p<0.01$ inflation versus inflation; all comparisons, ANOVA-SNK (two-tailed). acceptable during the periods of study. Arterial $\mathrm{Po}_{2}$ increased significantly during balloon inflations and this may have been related to favorable alteration of ventilation perfusion relationships lengthening of pulmonary transit time and/or reduced intracardiac right left shunting during the balloon inflations.

The LV performance and systolic function as described by developed peak systolic pressure, ejection fraction, and cardiac output was well maintained when the ascending aortic systolic pressure was elevated approximately $60 \%$ over baseline, even during $\beta$-adrenoceptor blockade (Figs. 4 and 5). Similarly, there was little evidence of $L V$ diastolic dysfunction. The premature LV at this stage of development is characterized by a relatively low myocardial mass as well as a lower ratio of contractile elements to supportive tissues, and some have suggested the newborn LV is relatively sensitive to increasing afterload (22). However, this observation was noted primarily when the LV was simultaneously volume loaded (22). Nevertheless, despite these developmental considerations, this study suggests that the preterm LV is capable of considerable reserve augmentation of developed pressure ( $60 \%$ over baseline) when preload is constant, and is capable of sustaining unchanged systolic and diastolic pump performance during these acute pressure loading conditions.

It is well-established that plasma catecholamines (EPI, NOREPI) increase markedly after clamping of the umbilical cord at birth (10). The baseline plasma catecholamine concentrations in our preparation were elevated well above fetal levels $(10,11)$. The basal circulating catecholamine levels were similar to those previously described in comparably treated lambs at similar gestational and postnatal age $(10,11)$. Although, plasma catecholamine metabolism may be altered by $\beta$-adrenoceptor blockade, in this study as in others, catecholamine levels did not change within $10 \mathrm{~min}$ after the administration of propranolol (20). However, plasma catecholamine levels increased significantly during both ascending aortic balloon inflations (before and after administration of propranolol). The balloon inflations were not associated with acidosis or hypoxia, and thus these variables could not have stimulated catecholamine secretion (4). Several studies have demonstrated intact and relatively increased carotid baroreceptor activity in fetal lambs at comparable gestation $(23,24)$. Itskovitz et al. (24) demonstrated baroreceptor activity in the fetal lamb by inflating a balloon catheter in the descending aorta-presumably distending the carotid sinus. In this study, downstream flow, pressure, and total systemic vascular resistance at 3-5 min inflation were maintained, and cardiac rate increased slightly despite balloon inflation and partial ascending aortic obstruction. It is possible that inflation of the balloon catheter in the ascending aorta was initially associated with a transient reduction of distension pressure in the transverse aortic arch and carotid sinus. The roles of carotid and aortic baroreflex control of sympathetic activity are well described (25). Such baroreflex activity may have contributed to the eventual hemodynamic "adjustments" observed after 3-5 min of balloon inflation. Inasmuch as circulating EPI is primarily derived from the adrenal gland in the preterm lamb, baroreceptor activation during the balloon inflation in the ascending aorta may have led to splanchnic sympathetic neural discharge and EPI secretion from the adrenal medulla $(13,23,26)$.

Sympathetic innervation of the preterm LV is relatively incomplete (12). Under these circumstances the performance of the preterm LV may be relatively dependent on circulating catecholamines that are increased during the transitional period after birth $(3,6,10,11)$. Indeed, various studies have demonstrated that the LV is relatively "hypercontractile" soon after birth and basilar cardiovascular performance is in part dependent on circulating epinephrine $(2-8,22)$. In this study a high level of LV contractility was observed soon (2-3 h) after birth when circulating catecholamines approach their greatest levels $(10,11)$. The data suggest that increases of circulating EPI contributed to the greater level of LV performance during acute pressure loading. 
This interpretation is further supported by the relatively high dose of propranolol required for $\beta$-blockade. The dose of propranolol $(3 \mathrm{mg} / \mathrm{kg}$ ) required to produce $\beta$-adrenoceptor blockade (defined by isoproterenol infusion) was 10-30 times that generally required for $\beta$-adrenoceptor blockade in mature animals and human beings $(20,27)$. Although with propranolol there was a marked decrease of chronotropy, after pacing there was no significant decrease of baseline LV contractility as manifested by mean velocity of circumferential fiber shortening ratio LVPSP:ESV, ventricular ejection fraction, or cardiac output at similar heart rates, preload, and afterload. Such dissociation between inotropy and chronotropy is frequently mentioned, but the mechanism remains unclear. $\beta$-Adrenoceptor blockade induces complex and opposite hemodynamic changes in the intact circulation (27). Changes of cardiac output and peripheral vascular tone may simultaneously stimulate reflex sympathetic "contractile" tone as well as changes of arterial and venous compliance (preload and afterload) which in turn influence pump performance. However, in this study heart rate was unchanged (paced) and LV preload and afterload were uninfluenced by propranolol. $\beta$-Adrenoceptor blocking agents may possess "intrinsic sympathomimetic activity" leading to dissociation of inotropy and chronotropy; however, such activity has not been observed with propranolol (28).

Alternatively, the apparent unchanged indices of LV performance and contractility after high dosage "competitive" $\beta$-adrenoceptor antagonist, propranolol, may have been related to the relatively high circulating levels of catecholamines soon after birth. Consequently, we subsequently attempted to study five similar preterm lambs using even higher doses $(5-10 \mathrm{mg} / \mathrm{kg})$ of propranolol. Unfortunately at these higher doses there was usually sudden and marked deterioration of the lambs associated with bradycardia (failure to pace) as well as profound arterial hypotension, and these findings cannot be differentiated from the "toxicologic" effects of propranolol. As in this study of preterm lambs, others have demonstrated that soon after birth of the full-term lamb, the LV is relatively hypercontractile when compared with that of the older lamb (7). Our observations differ from those of Clyman et al. (8) who demonstrated a decrease of "contractility" (peak DP/dt) after $\beta$-adrenoceptor blockade in surfactant treated preterm lambs with a patent ductus arteriosus (8). However, their preparation was studied at approximately 4$6 \mathrm{~h}$ after birth when reported mean plasma EPI concentration was approximately $50 \%$ of the mean concentration measured in this study.

We attempted to further characterize the LV contractile state by evaluating LV-PSP:ESV slopes and ratios under comparable conditions of pressure loading (baseline/inflation) both before and after propranolol. As mentioned, LV pump performance was well maintained during these inflations and heart rate and preload were essentially unchanged. A shift of the slope of the LV end-systolic pressure:ESV relationship or change of the ratio LV-PSP:ESV is an accepted descriptor of contractile state (21). We did not use arterial end-systolic pressure, as the dicrotic notch was not reliably recorded during balloon inflation. However, the use of LV-PSP reflects alterations of afterload and contractility in the intact circulation and is particularly useful for comparison of serial studies at the same loading conditions in the same animal $(7,21)$. Our initial calculations suggested no significant shift of the left ventricular peak systolic pressure endsystolic volume relationship, i.e. there was no apparent decrease of contractility after propranolol. This occurred because there was no significant increase of left ventricular end-systolic volume, as would be expected after balloon inflations, despite the markedly increased LV peak systolic pressure. In fact, in several instances the LV-ESV actually decreased during balloon inflations despite the greater LV-PSP during inflation. We recognized the limitation of constructing the LVP-PSP:ESV relationship from the limited data points (baseline/inflation), furthermore the latter observations and catecholamine data suggested that contractile state may have changed during balloon inflation. Consequently, we compared the ratios of LV-PSP:ESP at baseline and during inflation (Table 2). The ratios increased significantly during inflation; i.e. LV-ESV became relatively less for the given level of pressure. These findings suggest that an increase of left ventricular contractility occurred during the balloon inflations (21). This change in contractile state was associated with dramatically increased concentrations of circulating catecholamines and was partially blunted by propranolol. This "partial" $\beta$ adrenoceptor blockade may be related to the high circulating levels of competing catecholamine or alternatively might be related to $\alpha$-adrenoceptor stimulation by EPI and NOREPI (29). Thus, an increase of LV contractility apparently contributed to the ability of the preterm LV to augment developed pressure (at similar heart rate and preload) as well as maintaining unchanged systolic and diastolic pump performance.

Finally, it is recognized that the increased catecholamine secretion and presumably altered contractility after balloon inflation represents a limitation of this study, because it would have been preferable to maintain a "constant" contractile state. Our findings confirm that such experimental perturbations may alter plasma catecholamine secretion and influence the "steady" state of LV contractility. Clearly, one cannot describe contractile state using a "continuous" relationship of peak-systolic (or end-systolic) pressure on volume under such circumstances. Others (24) have suggested that such limitations should be considered in studies of contractility where alterations of preload or afterload might potentially alter baroreceptor discharge and catecholamine secretion.

In summary, our data suggest that the preterm LV possesses considerable reserve capacity in response to acute pressure loading in the first 3-4 h after birth. From the developmental point of view, this reserve capacity represents a potentially favorable adaptation to the acute alterations of increasing left ventricular afterload associated with parturition, removal of the placenta, and closure of the ductus arteriosus (30). This capacity complements the volume loading reserve of the preterm LV as well (9). Furthermore, we have observed that soon after birth the preterm LV appears to function at a very high level of contractility, and LV inotropy was relatively unchanged even in the face of high dose pharmacologic $\beta$-adrenoceptor blockade. In addition, the preterm LV appears to be capable of integrating augmented catecholamine secretion, with an increased state of contractility at rest and during ascending aortic pressure loading. Thus, basal pump function as well as volume and pressure loading reserve may be supported in part by augmented secretion of catecholamines postnatally $(8,9,11,13,29)$. These considerations are relevant to the successful adaptation of the LV to postnatal transitional circulatory events associated with increased afterload as well as to certain forms of obstructive congenital cardiac lesions or shunts which are associated with reduction of $\mathrm{LV}$ output.

Acknowledgement. The author thanks Tina M. Gingrich for help in preparation of this manuscript.

\section{REFERENCES}

1. Klopfenstein HS, Rudolph AM 1978 Postnatal changes in the circulation and responses to volume loading in sheep. Circ Res 42:839-845

2. Reimenschneider TA, Brenner RB, Mason DT 1981 Maturational changes in myocardial contractile state of newborn lambs. Pediatr Res 15:349-356

3. Geis WP, Tatooles CJ, Priola DV, Friedman WF 1975 Factors influencing neurohumoral control of the heart in the newborn dog. Am $\mathrm{J}$ Physiol 228:1685-1689

4. Lee JC, Werner C, Downing SE 1980 Adrenal contribution to cardiac responses elicited by acute hypoxia in piglets. Am J Physiol 239(8):H751-H755.

5. Downing SE, Lee JC 1983 Analysis of cardiac adrenergic mechanisms in hypoxic lambs. Am J Physiol 244:H222-H227

6. Erath HG, Boerth RC, Graham TPJ 1982 Functional significance of reduced sympathetic innervation in the newborn dog. Am J Physiol 243:H20-H26

7. Teitel DF, Sidi D, Chin T, Brett C, Heymann MA, Rudolph AM 1985 Developmental changes in myocardial contractile reserve in the lamb. Pediatr 
Res 19:948-955

8. Clyman RI, Teitel D, Padbury J, Roman C, Mauray F 1988 The role of $\beta$ adrenoceptor stimulation and contractile state in the preterm lamb's response to altered ductus arteriosus patency. Pediatr Res 23:316-322

9. Baylen BG, Ogata H, Ikegami M, Jacobs H, Jobe A, Emmanouilides GC 1986 Left ventricular performance and contractility before and after volume infusion: a comparative study of preterm and full-term newborn lambs. Circulation 73:1042-1049

10. Padbury. JF, Diakomanolis ES, Hobel CJ, Perelman A, Fisher DA 198 Neonatal adaptation: sympatho-adrenal response to umbilical cord cutting. Pediatr Res 15:1483-1487

11. Padbury JF, Polk DH, Newnham JP, Lam RW 1985 Neonatal adaptation: greater sympathoadrenal response in preterm than full term fetal sheep at birth. Am J Physiol 248:E443-E449

12. Lebowitz EA, Novick JS, Rudolph AM 1972 Development of myocardial sympathetic innervation in the fetal lamb. Pediatr Res 6:887-893

13. Padbury J, Agata Y, Ludlow J, Ikegami M, Baylen B, Humme J 1987 Effect of fetal adrenalectomy on catecholamine release and physiologic adaptation at birth in sheep. J Clin Invest 80:1096-1103

14. Jobe A, Ikegami M, Glatz T, Yoshida Y, Diakamanolis E, Padbury J 1981 The duration and characteristics of treatment of premature lambs with natural surfactant. J Clin Invest 67:370-375

15. Clyman RI, Heymann MA, Maurray FM 1987 How a patent ductus arteriosus affects the premature lambs ability to handle additional volume loads. Pediatr Res 22:531-535

16. Baylen BG, Ogata H, Oguchi K, Ikegami M, Jacobs H, Jobe A, Emmanouilides GC 1985 The contractility and performance of the preterm lamb left ventricle before and after early patent ductus arteriosus occlusion in surfactant treated lambs. Pediatr Res 19:1053-1058

17. Quinones MA, Gaasch WH, Alexander JA 1976 Influence of acute changes in preload, afterload, contractile state and heart rate on ejection and isovolumic indices of myocardial contractility in man. Circulation 53:293-301
18. Sandler H, Dodge HT 1968 The use of single plane angiocardiograms for the calculation of left ventricular volume in man. Am Heart J 74:325-334

19. Baylen BG, Ogata H, French WJ, Emmanouilides GC 1984 Accurate internal correction for magnification of cineangiocardiography in infants with congenital heart disease. Am Heart J 107:113-118

20. Hjemdahl P, Akerstedt T, Pollare T, Gillberg M 1983 Influence of beta adrenoceptor blockade by metoprolol and propranolol on plasma concentrations and effects of noradrenaline and adrenaline during I.V. infusion. Acta Physiol Scand [Suppl] 515:45-53

21. Sagawa K 1981 The end systolic pressure-volume relationship of the ventricle: definition, modifications and clinical use. Circulation 63:1223-1227

22. Friedman WF 1972 The intrinsic physiologic properties of the developing heart. Prog Cardiovasc Dis 24:87-111

23. Itskovitz J, LaGamma EF, Rudolph AM 1983 Baroreflex control of the circulation in chronically instrumented fetal lambs. Circ Res 52:589-596

24. Blanco CE, Dawes GS, Hanson MA, McCooke HB 1988 Carotid baroreceptors in fetal and newborn sheep. Pediatr Res 24:342-346

25. Sander JS, Ferguson DW, Mark AL 1988 Arterial baroreflex control of sympathetic nerve activity during elevation of blood pressure in normal man: Dominance of aortic baroreflexes. Circulation 7:279-288

26. Graham ADM, Longo LD, Cheung CY 1986 Catecholamine secretion from the adrenal medulla of the fetus, regulation by hormones. $J$ Dev Physiol 8:227-235

27. Taylor SH 1986 Beta-blocking drugs and myocardial function. J Cardiovasc Pharmacol 8:575-582

28. Prichard BNC, Tomlinson B 1986 The additional properties of $\beta$-adrenoceptor blocking drugs. J Cardiovasc Pharmacol 8(suppl 4):S1-S15

29. Cheng JB, Carnett LE, Goldfein A, Roberts JM 1980 Decreased concentration of myocardial $\alpha$-adrenoceptors with increasing age in the foetal lamb. $\mathrm{Br} \mathrm{J}$ Pharmacol 70:515-517

30. Rudolph AM 1970 The changes in the circulation after birth. Their importance in congenital heart disease. Circulation 41:343-359 